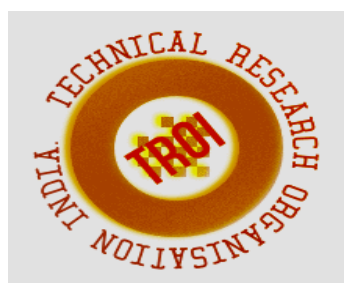

\title{
SECURE ATM USING NFC AND ADAPTIVE AUTHENTICATION
}

\author{
Sophia S. ${ }^{1}$, Indhumathi R S ${ }^{2}$, Divya $\mathrm{S}^{3}$, Divyashree $\mathrm{V}^{4}$, Dharshan Shankar $\mathrm{S}^{5}$ \\ ${ }^{1}$ Assistant Professor, ${ }^{2,3,4,5}$ UG Students, \\ Department of Computer Science and Engineering \\ Dr. T. ThimmaiahInstitute of Technology, Karnataka, India- 560100 \\ 1'sophia7selvaraj@gmail.com, 2indhushyla1997@gmail.com, \\ 3divyas11223@gmail.com, 4divyacharya97@gmail.com, \\ 5dharshan.ttit@gmail.com
}

\begin{abstract}
ATM has become a most used means for to its users.
\end{abstract} improved levels.

Multifactor people to withdraw money from their bank which modify itself according to the risk profile of accounts, but ATM Cards are no longer secure the user. Risk profile is formed by comparing the because a skimmer can be fixed on ATM usersbehavior such as login time, location, Device machine and the account details can be easily type, Number of failed attempts, the amount tin hacked . The thesis provides the development the user account, Risk level/score will be of Multi-Factor authentication along with generated by using Risk engine that is integrated Near Field communication, where Multi-factor with the risk based authentication system. And the authentication includes the design of risk proposed system works based on the Machine engine proportion with the system to check the learning Algorithms and Adaptive authentication. users past login records and generate suitable Machine learning algorithms are trained to learn pattern using machine learning algorithm and patterns from existing data and predict the calculate the risk score, based on the user risk unknown value when provided with the new set profile it provides different means of of data.And Adaptive authentication is a method authentication. Thus the adaptive for selecting the right authentication factors authentication helps in providing high-security depending on a usersrisk profile. By considering

the Risk profile of the user, he/she is been

Keywords: Near Field Communication (NFC), challenged with different authentication methods. Risk based authentication, Adaptive Any how the actual user need not required to pass authentication multiple factors of authentications to prove his identity, while a suspicious user needs to pass all authentication methods he is required with, this ensures high security to its users. The remaining paper is arranged in the following manner- Section 2 briefly tells about the research works, enlancingtheir advantages and disadvantages. Section 3 elaborated the detailed structure of proposed method. Experimental results are depicted in Section 4 and Section 5 presents the conclusion.

\section{LITERATURE SURVEY} Moreover, passwords/PIN are simply knowledge basedinformation that can be shared amongst users that led to a major drawback of Single-Factor authentication. Thus, two factor authentication or Muti-Factor authentication is preferable due to its tudies have been done on secure ATM Different studies have been done on secure ATM authentication having its own advantages and 
disadvantages. The Naïve Bayes classifier contextual information, requested services and algorithm adopts conditional independence; it the type of transaction.

consider that an attribute value of a given class is independent values of other attributes.

\section{PROPOSED WORK}

Nagaratna et al. [1] has suggested multiple bank The proposed section presents the systems set up accounts into single ATM card using finger print and detailed explanation of essential processes based authentication and face recognition which involved. The implementation of secure ATM provides high security. The hardware used in the system uses two new methodology:

system are mobile phone, face recognition, fingerprint scanner. Inputs are given by user and its verified with the previously stored data in the database and as output the user is authenticated.

Anirudhanadukkathayar [2] proposed 4-digits PIN as the knowledge factor, and NFC enabled smartphone, the input like 4-digit pin number and NFC card is given by the user for process of authentication. If the NFC card is valid the the user is allowed to enter the 4-digit pin number. This pin is verified for the process of authentication.

Anusha mandalapuet al. [3] describes three factor authentication scheme employing NFC, Dash matrix algorithm and one timepassword in this three factor Authentication. user can also block his ATM card if itsmissed using negative pattern which is already registered when the user create the account.

Dasgupta et al.[4] implemented a design for selecting factors dynamically for multifactor authentication. Trustworthy values were calculated for each factor using mathematical objectives functions. This selection of factors was affected by the device, media and external conditions like noise and light. This techniques reduced the repetitive selection of the same set of authentication factors.

Akio Ogiharaet al. [5] implemented a system using biometrics. It is performed by using 10 types of biometric feature of hand shape, which are extracted from key strokes provided in an ATM operation. Moreover, it calculates the similarities between current ATM operator and genuine user in consideration of key- press timing. The components used in this systemsare biometrics sensors which are embedded in ATM Machine.

Diep et al. [6] present a mathematical risk based techniques called multifactor evaluation process that assigned numerical weights to risk elements based on confidentiality, integrity and availability of the outcomes. The risk is measured based on

1)NFC

2)Risk Score Authentication.

An overview of the system is shown in Figure.1

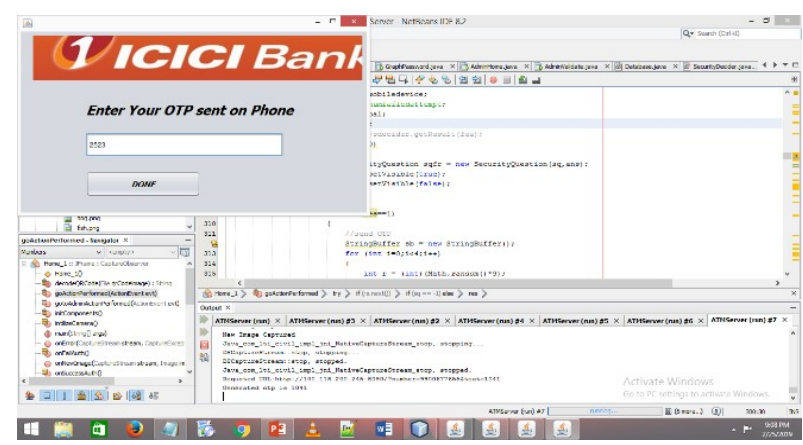

Figure 1:System Overview

The process aims at reliable dealings with banks and ensures ease of use for ATM users. This process is further divided as follows:

\section{A.NFC Card Validation}

System instead of ATM card, NFC enabled mobile phone can be used to login to ATM. With the use NFC there is no need for swiping. The user is required to tap the cell phone on the NFC tag which is fixed on the ATM. Upon successful NFC tagging, a webpage on the Cell phone's browser requests for a pre-registered phone number and PIN as a user input. When user provides it, the user phone number and PIN is sent to ATM Server. It is used to validates the NFC card access and if it is a valid card, it passto the next step of authentication.

\section{B. Risk score calculator}

Risk score calculator is a core component of our system which can predict the risk level of a user. In this paper, the risk score calculator is designed using a Naïve Baiyesclassifier which is a machine learning algorithm.

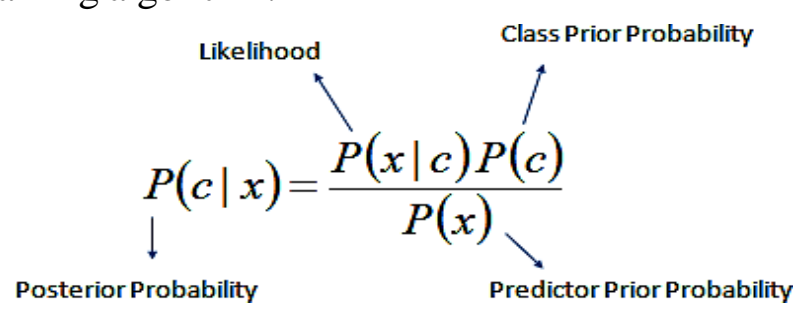


Further the calculator is divided as follows:

Score Training: This module trains the Naïve Baiyesclassifier to find the risk score for the user account based on the access attributes and user attributes.

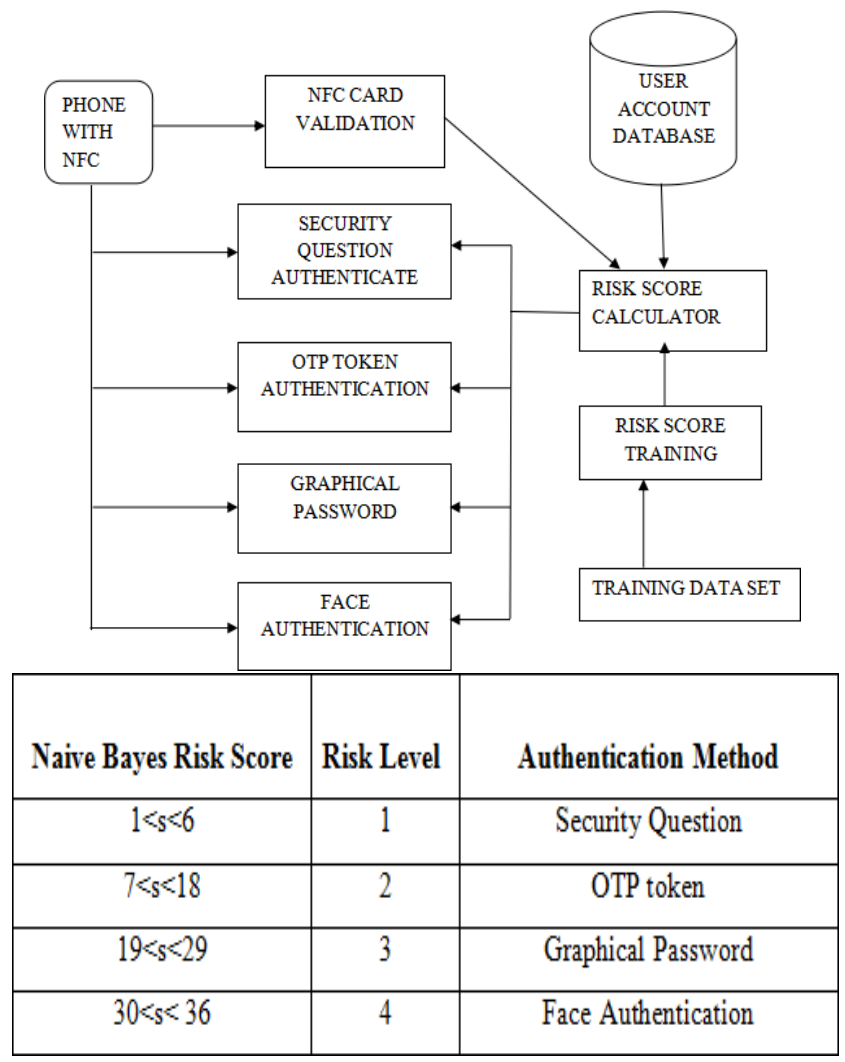

Risk Score Calculator: This module uses the trained Naïve Baiyesclassifier to calculate the risk score and based on calculated risk score, it chooses the authentication method.

Below Table I represent the Risk score value and corresponding authentication method.

\section{Table I Risk Score Value}

Security Question Authentication: This method provides a security question to user which user has registered during the creation of account and as verifies the user answer. Based on the results allows the user to transaction stage.

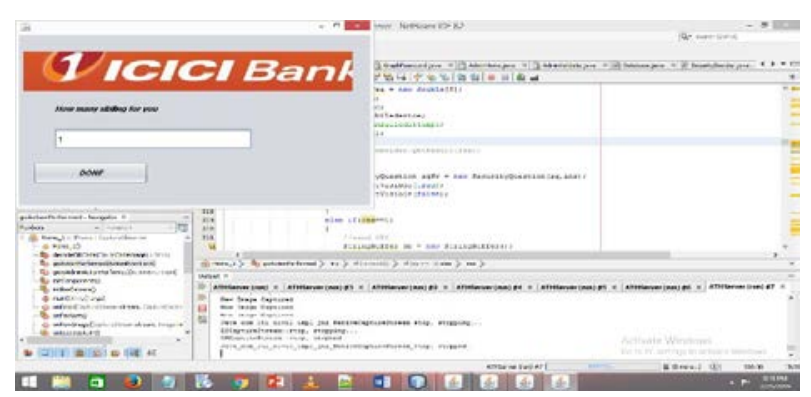

Figure 2: Security Question
OTP Token Authentication: A four digitOneTime Password (OTP) is generated on the mobile screen which is valid only for few seconds, say 60seconds asinFigure2.If it exceeds the timeout, then a OTP is regenerated. The user wantto enter the same OTP on ATM keypad. If its correct OTP token, it allows user to transaction stage.

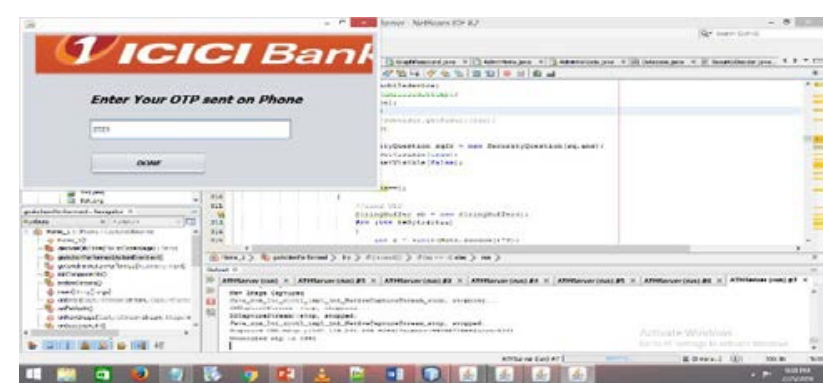

Figure 3: One Time Pad

Graphical Password: User registers graphical password in form his favorite animals, vegetables, fruits in a series, The pictures are shown and user must choose the correct order as he registered. If the selection is correct, it allows the user to transaction stage.

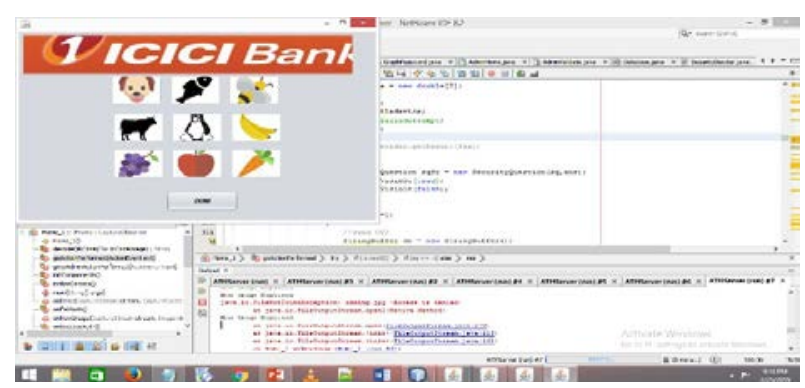

Figure 4: Graphical Questions

Face Authentication: This method captures the face image and compares to the registered face image, if it is similar, it allows the user to transaction stage.

When the user enters to the a ATM the user should tap his mobile phone on the NFC tag. If the NFC card is validated then user is allowed for further process. Then the risk engine is activated and the user is asked for some of security questions, suchas security question authentication, OTP Token Authentication, graphical password, face authentication according to risk score value if conditions satisfies then the user is authenticated user and he is allowed for further process, and allowed for transaction. The risk score value is calculated using some of the 
user behavior which considered as the training data set and this is stored in database.

Following parameters is used as test cases

1. Login time

2. ATM Location

3. Mobile Device

4. No of failed attempts

5. Amount in the Users account

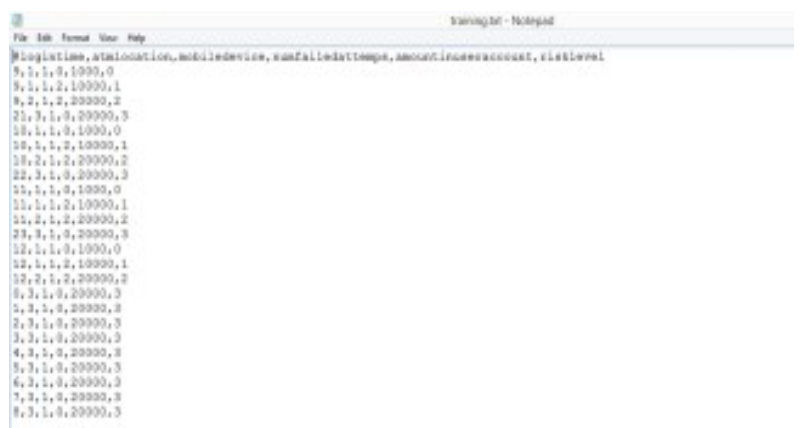

\section{Figure : 5 Naïve Bayes}

The below Figure 6 represents Flow Chart of Secure ATM. When the user enter the ATM firstly it will collect the NFC informationrom the user and it will check whether the card is valid or invalid. If the card is invalid it again ask user for proper NFC information card. If the card is valid it moves to further process, it will collect the user attributes such as login time, OS version, location of ATM, Amount present in user account and it will access the attributes. Now the risk engine is initiated for calculating the risk score.

According to the risk score.

1)If the risk score value is $r<6$ then it will ask for some security questions.

2) If the risk score value is $6<r<18$ then it will ask for OTP token.

3)If the risk score value is $18<r<29$ then it will ask for some graphical password.

4) If the risk score value $29<\mathrm{r}<36$ then it will ask for face image of user.

If the authentication is success it will allow for transaction else it will display an error and process is terminated.

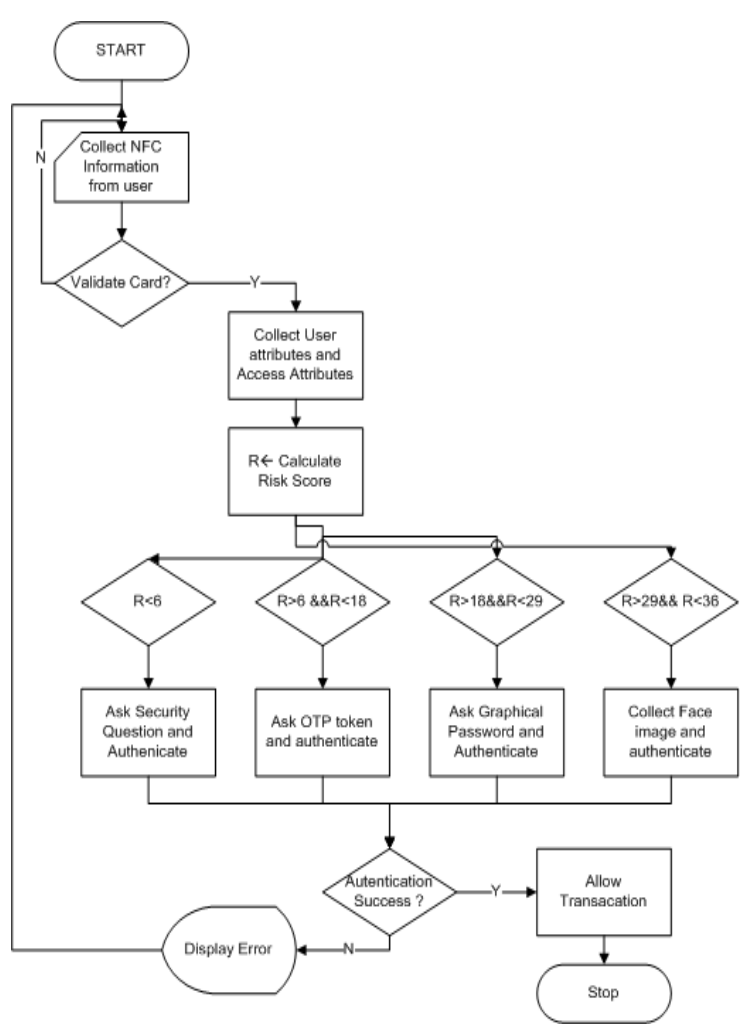

Figure 6 : Block Daigram of Secure ATM

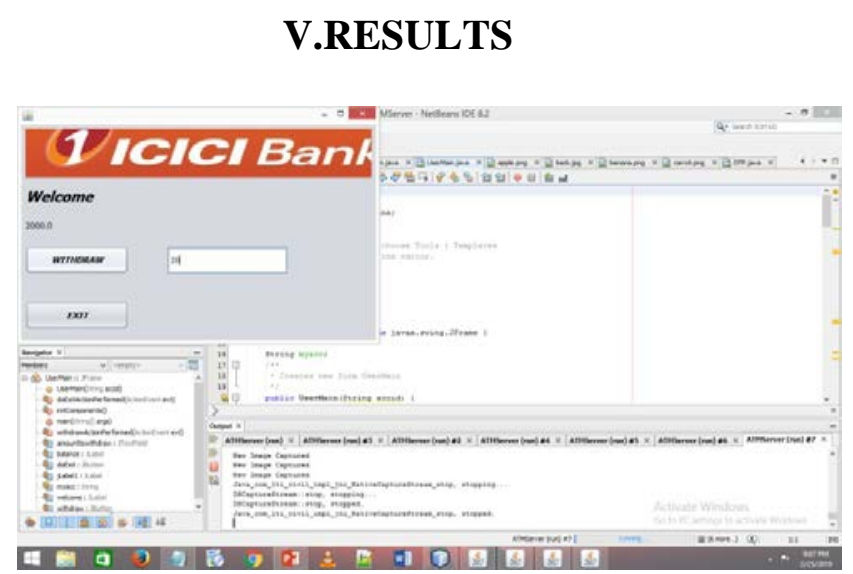

\section{CONCLUSION}

The ATM Electronic Transaction is playing very persistent and pervasive role in the present world. The ATM should be made up of robust infrastructure setup to with stand any kind oftheft. In Existing System authentication and authorization is done by using credit card and pin number. It leads to low level of authentication and leads to theft . Hence developed new method called Multi factor authentication using two methodology NFC (Near Field Communication) and Risk Based Authentication. The Benefits of NFC are avoiding skimming of cards, it is easyaccessible to user and reliable, Feasible and Benefits of Risk Based 
Authentication provides High Level security and it provides risk level questions based on user behavior. In this paper it mainly providehigh level of security, avoid bank accounts from different types of theft and attacks, and avoid the usage or Carrying of multiple ATM Card.

\section{REFERENCES}

[1] Nagaratna,"Highly Secure Multiple Account Bank Affinity Card- A Successor for ATM Card”, IEEE Publisher, 2015

[2] anirudhanadukkathayaer," secure multifactor authentication payment system using nfc",10th International Conference on Computer Science \& Education (ICCSE 2015) July22-24, 2015. Fitzwilliam College, Cambridge University, UK [3] Anusha Mandalapu, ”AnNFC featured three level authentication system for tenable transaction and abridgment of ATM card blocking intricacies”, IEEE 2015.

[4] Dipankar Dasgupta, Abhijit Nag, and Arunava Roy,"Toward the design of adaptive selection strategies for multi-factor authentication”. Computers and security, pp,2016.

[5] Dipankar Dasgupta, Abhijit Nag,andArunavaRoy, " Toward the design of adaptive selection strategies for multi-factor authentication”. Computers and security,2016.

[6] DiepN.N.,Y.-KLee, HJ.Lee, S.Lee, "Contextual Risk-based Access control”, Security and management,2007 